\title{
Staged Thermomechanical Testing of Nickel Superalloys Produced by Selective Laser Melting
}

Zhengkai Xü ${ }^{a}$, C. J. Hyde ${ }^{a}$, A. Thompson ${ }^{a}$, R. K. Leach ${ }^{a}$, G. A. Ravi ${ }^{b}$, I. Maskery ${ }^{a}$, C. Tuck $^{\mathrm{a}}$, A. T. Clare ${ }^{\mathrm{a}^{*}}$

a. Department of Mechanical, Materials and Manufacturing Engineering, University of Nottingham, University Park, NG7 2RD, United Kingdom

b. Renishaw PIc, AMPD, Brooms Road, Stone, Staffordshire, ST15 0SH, United Kingdom

Corresponding author: adam.clare@nottingham.ac.uk

\section{Abstract}

The creep performance of additively manufactured components remains an issue before additive manufacturing can be put fully implemented. In this study, Inconel 718 two-bar specimens are produced by selective laser melting and subjected to a 'staged' creep test. Creep test was interrupted at critical junctures and X-ray computed tomography measurements performed at various extensions of the specimen. Periodic $X$-ray computed tomography measurements allow, for the first time, examination of the specimens during creep testing. Evaluation of specimen performance shows the number and size of pores within the specimen increasing over time as a result of classical creep mechanisms. Location and tracking over time of weak points are performed, allowing early estimation of sample failure points. This information is valuable to selective laser melting practitioners who seek to optimise the build strategy in order to minimise in-built defects.

Keywords: Creep, Two-Bar Specimen, X-ray Computed Tomography, Selective Laser Melting, Mechanical Testing, Nickel Superalloys

\section{Introduction}

Selective laser melting (SLM) is a powder bed fusion based additive manufacturing (AM) technique. It is a near-net-shape technique which provides a high flexibility in producing complex geometrical features in high value components such as turbine blades. However, when considering the mechanical testing of nickel superalloys 
processed by SLM, the published work is limited to relatively simple tests, e.g. hardness and tensile testing. For example, Amato et al. [1] studied the effects of the build orientation and post-treatments on the hardness and tensile properties of Inconel 718 samples built in two different atmospheres. They confirmed that variations in the mechanical properties were mainly caused by the $\gamma$ " precipitates. Jia et al. [2] investigated the effects of changing SLM build parameters on Inconel 718 samples with an examination of densification, hardness and wear performance. An optimum energy density which resulted in improved mechanical properties was determined. However, fewer researchers have examined the more complex failure mechanisms of SLM samples, such as creep or fatigue behaviour. Rickenbacher et al. [3], for example, not only studied tensile properties of SLM fabricated IN738LC, but also demonstrated that the creep performance of that material was inferior compared to conventionally manufactured samples. In the study performed by Pröbstle et al. [4], however, Inconel 718 SLM samples were found to possess improved creep strength over cast and wrought samples. Pröbstle et al. also showed that post-process heat treatment further improved the creep performance of Inconel 718 , although fracture results were not included.

Of the aforementioned mechanical properties, creep resistance is a key mechanical property for components deployed in high-temperature applications [5], and the use of additively manufactured parts here has so far been limited by the poor creep performance. This is largely based upon the assumptions on the evolution of 'in-built' defects at high temperatures. Analysis of the response of AM components to creep is therefore of critical importance, particularly the response of common defects produced during the AM processes, which are expected to evolve under creep conditions. It is well known that creep is a time-dependent phenomenon, but current conventional testing methods do not examine the entire sample or provide direct evidence of defect evolution during creep testing. In this paper, a method for studying such defect evolution is presented. Through the use of X-ray computed tomography (XCT)[6], defects can be non-destructively detected allowing the measurement procedure to continue. In a previously reported study [7], XCT measurement was applied to detect defects in a sample before and after fatigue fracture. Babout et al. [8] studied the damage mechanisms of room temperature tensile testing of powder metallurgy fabricated samples, the entire testing procedure was divided into seven deformation 
stages and XCT measurement was performed in each stage. In another study [9], an in-situ XCT measurement was applied to study a high-temperature tensile testing, the evolution of samples' deformation was clearly demonstrated. In this study, XCT measurement is undertaken at periodic stages of the creep test. Through comparison of different XCT datasets, the evolution of the defects can be characterised. XCT has so far been used for non-destructive testing to measure the spatial distribution of pores in SLM structures in recent research, and a review of such studies is presented by Thompson et al. [10]. Specifically, Maskery et al. [11] successfully quantified and characterised the porosity in SLM built structures. Maskery et al. demonstrated the feasibility of using XCT to give a sufficient description of part porosity, pore size and pore shape. In another study [12], XCT was applied to locate and measure the defects in samples built by selective electron beam melting (SEBM). The size, volume fraction and spatial distribution of these defects were well recognised and characterised. Particularly, a voxel size of $9.9 \mu \mathrm{m}$, which gives a minimum detectable pore equivalent diameter of approximately $25 \mu \mathrm{m}$, was found adequate to locate all large-scale defects in the sample.

The novel approach presented in this work combines staged creep testing with XCT and provides new information regarding time-dependent creep phenomena. This approach also provides a new understanding of the creep performance of SLM processed Inconel 718.

\section{Experimental Methodology}

Inconel 718 two-bar specimens (TBSs) were subject to creep testing in this study. A TBS (as shown in Fig. 1(b)) is a specimen which designed to obtain information about both uniaxial creep strain rate and fracture life [13]. The raw TBS block (as shown in Fig. 1(a), in which the arrow indicates the SLM building direction) was built in a Renishaw AM250 laser melting system. The raw block was post-processed using a computer numerical controlled (CNC) milling machine to the specified dimensions (as shown in Fig. 2), defined by Hyde et al. [13]. Testing was carried out under a uniform tensile stress of $747.45 \mathrm{MPa}$ at a temperature of $650{ }^{\circ} \mathrm{C}$. These parameters were chosen to match a creep study made by Sugahara et al. [14] on standard wrought Inconel 718 samples. 

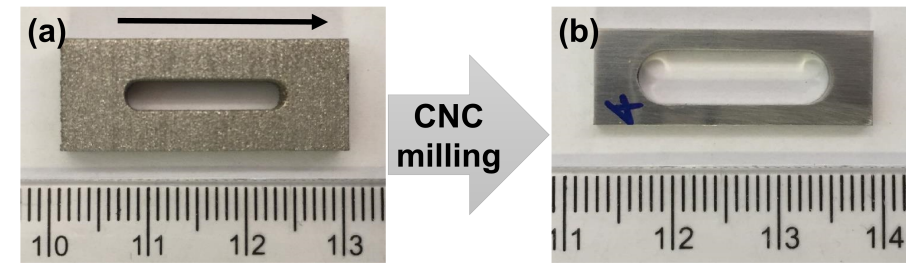

Fig. 1 (a) Raw TBS block (arrow indicates the SLM building direction); (b) CNC machined asbuilt TBS (dimensions in $\mathrm{mm}$ )

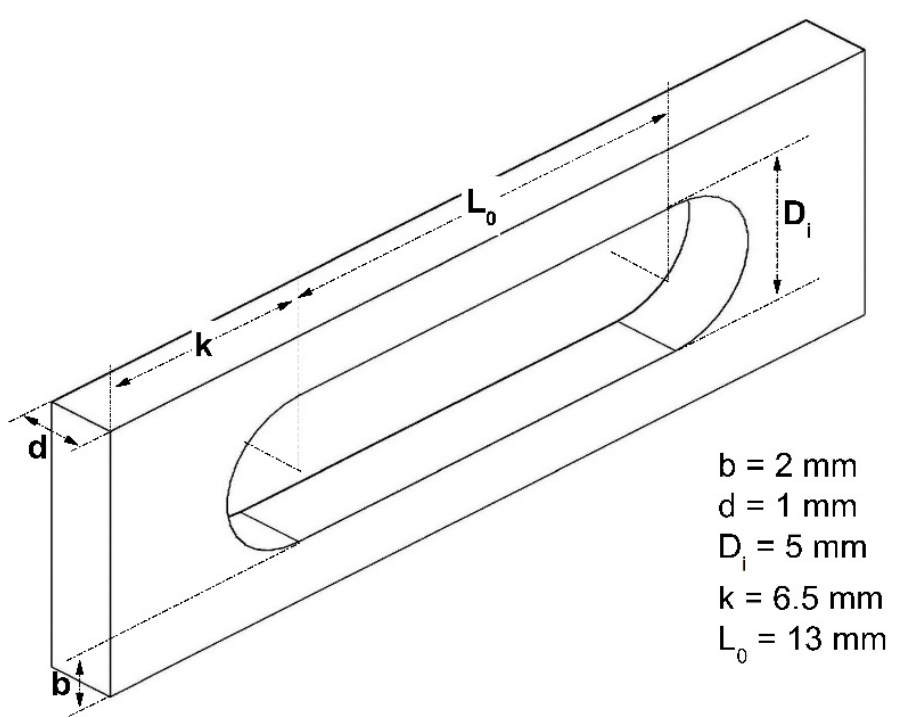

Fig. 2 Dimensions of the TBS used in this study [13]

Preliminary, uninterrupted, creep testing was carried out under the aforementioned conditions to estimate the extension range of the TBS. The extension and lifetime of four repeat samples obtained during preliminary testing are shown in Table 1.

Table 1

The extension and lifetime results of the preliminary testing

\begin{tabular}{cccc}
\hline Sample & $\begin{array}{c}\text { Initial elastic } \\
\text { extension / mm }\end{array}$ & $\begin{array}{c}\text { Failure extension / } \\
\mathrm{mm}\end{array}$ & Lifetime / s \\
\hline Sample A & 0.999 & 2.014 & 24240 \\
Sample B & 0.470 & 1.829 & 32880 \\
Sample C & 0.729 & 2.074 & 47760 \\
Sample D & 0.640 & 1.856 & 21960 \\
Average & 0.710 & 1.943 & 31710 \\
\hline
\end{tabular}

Based on the preliminary results, a strategy for staged creep testing was devised and is shown in Fig. 3. Testing was interrupted when the creep strain reached each pre- 
set extension. XCT measurements were then performed at these intervals as well as prior to testing and after failure, yielding four XCT datasets in total.

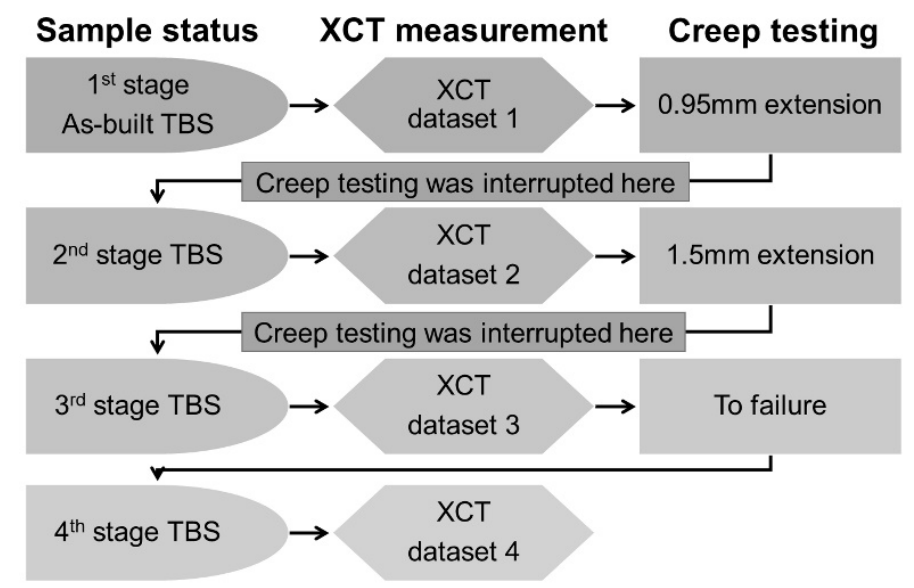

Fig. 3 Testing strategy for staged creep testing

A Nikon MCT 225 was used to perform the XCT measurements. Scan parameters were kept constant between measurements, using the following setup: source voltage $225 \mathrm{kV}$, source current $44 \mu \mathrm{A}$, exposure $2000 \mathrm{~ms}$ and geometric magnification 20x; yielding a reconstructed voxel size of $10 \pm 0.2 \mu \mathrm{m}$ (uncertainty taken from positional variability along the magnification axis between scans). The reconstructed voxel size limits the minimum detectable pore size, as it is generally accepted that the minimum detectable pore size is approximately that of a few voxels. In this study, the largest pores were predicted to be most likely to cause failure of the TBS, and so pores below the resolution of the system in the setup used were not considered. X-ray projections were formed from an average of two frames per projection. A warm up scan of over one hour was performed prior to each scan to minimise the effects of X-ray source fluctuation as the source temperature stabilised, and a $1.75 \mathrm{~mm}$ copper X-ray pre-filter was used. X-ray imaging and volumetric reconstruction were performed using Nikon software (Inspect-X and CT-Pro, respectively), using a filtered back projection reconstruction algorithm with a third order beam hardening correction and a ramp noise filter [15].

Analysis of XCT data was performed on TIFF images outputted in the arrangement shown in Fig. 4, considering only the parallel sections of the sample. The centre of the uniaxial section is set as the zero point; the positive and negative directions are marked in Fig. 4. All descriptions in the following are based on this convention. 


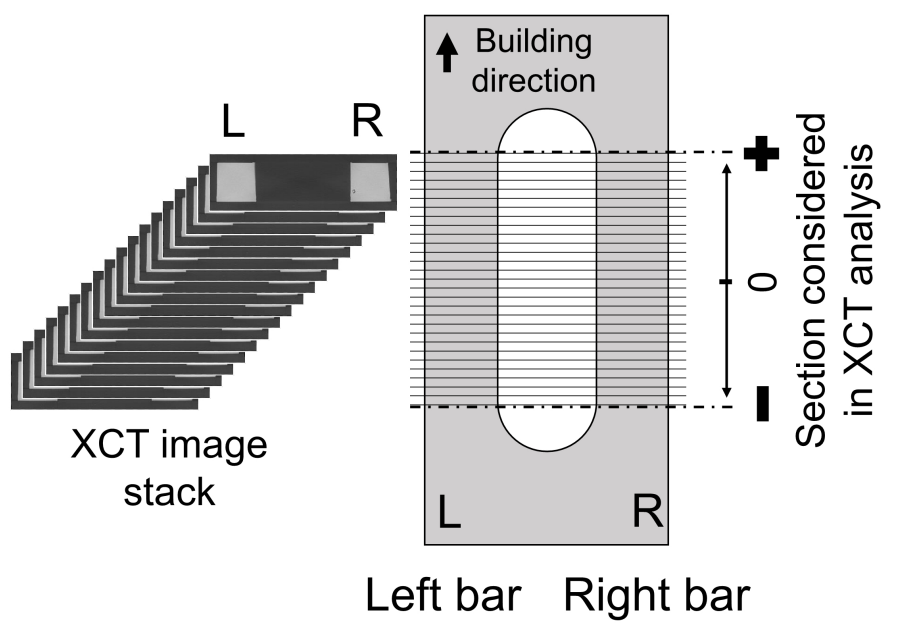

Fig. 4 XCT image stacking and the convention for referencing scanned position

Image stack analysis was performed using a combination of MATLAB [16] and ImageJ [17]. The ISO 50 surface determination method [18] (described diagrammatically in Fig. 5) was initially applied to threshold image stacks. However, in this case, the threshold ISO 50 grey value (as shown in Fig. 5(a)) provided clearly spurious thresholding results (as shown in Fig. 5(b)). The thresholding value was manually adjusted to ISO 78.6 (as shown in Fig. 5(a)) to provide a clear representation of pores in a number of example images while minimising noise in the binarised images (shown in Fig. 5(b)). The ISO 78.6 threshold was then applied to all images individually using MATLAB. The binary images produced by MATLAB were then analysed using Image J to calculate porosity, pore size and standard distribution metrics. In order to filter any remaining noise and focus only on the largest pores, areas larger than 20 pixels (which is five times larger than that used in previous work [8]) in the binarised images were treated as pores. This area is five times larger than that used in previous work [11], but was chosen in order to ensure complete noise filtration for comparison between test stages. This filtration is necessary as determining between the smallest pores and noise is highly complex, and can reduce the robustness of calculated porosities, which is essential for comparison purposes. 

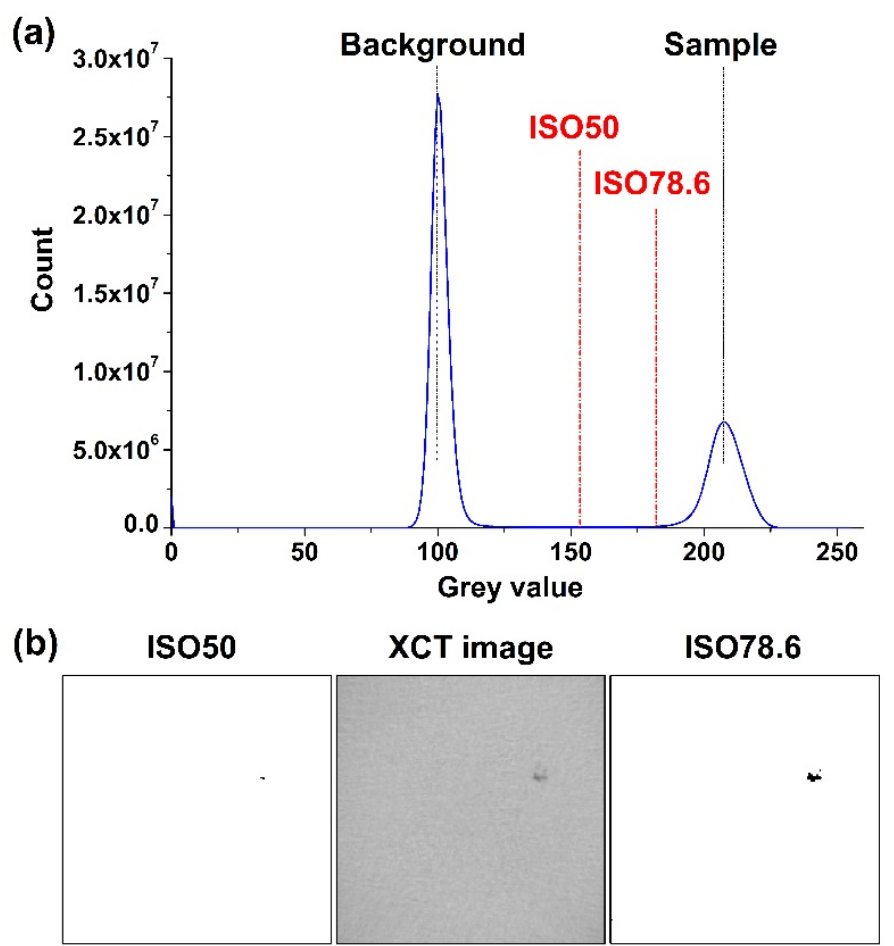

Fig. 5 Methods for thresholding XCT datasets, (a) The grey scale histogram of a XCT image, (b) XCT image and binarised images processed using ISO 50 and ISO 78.6 threshold values

A Hitachi TM3030 scanning electron microscope (SEM) was used to examine the fracture surfaces of creep samples (shown in Fig. 6).
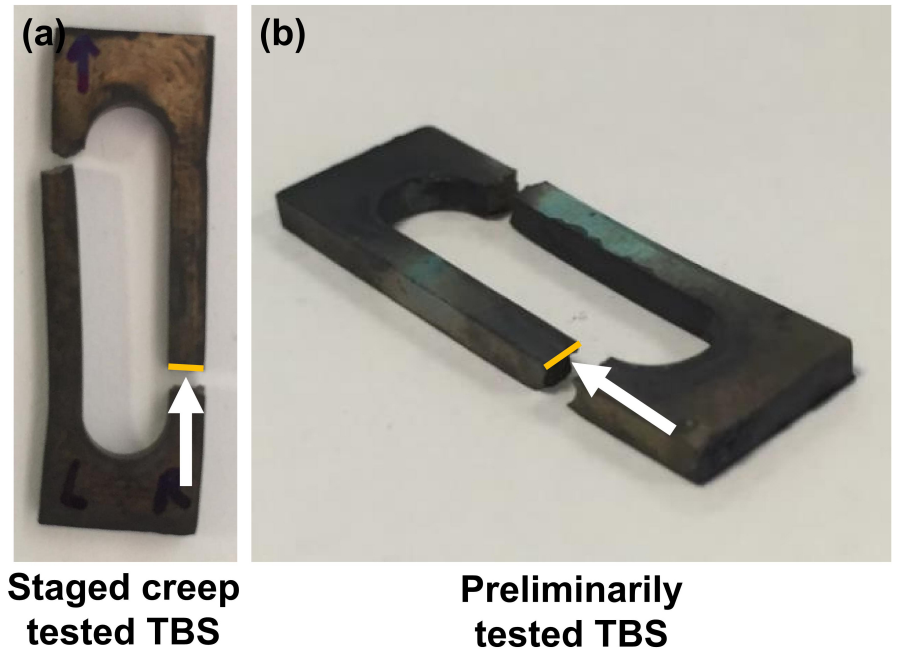

$$
\begin{gathered}
\text { Preliminarily } \\
\text { tested TBS }
\end{gathered}
$$

Fig. 6 (a) SEM observation strategy in staged tested TBS; (b) preliminarily tested TBS. Arrows indicate the SEM observation direction 


\section{Results}

\subsection{Staged creep testing results}

Table 2 shows the results of the staged creep testing. The extensions observed in the uniaxial section were found to be smaller than the pre-set extensions. The full lifetime of the staged tested TBS was 30\% greater to the average (listed in Table 1) obtained in the preliminary tests, but still in a reasonable range. However, the $2^{\text {nd }}$ and $3^{\text {rd }}$ stages have short experimental time which was effectively high-temperature tensile testing instead of creep. The $4^{\text {th }}$ stage was a creep test.

Table 2

Results of all three stages of the staged creep testing

\begin{tabular}{cccc}
\hline & $\begin{array}{c}\text { Pre-set } \\
\text { extension } / \mathrm{mm}\end{array}$ & $\begin{array}{c}\text { Real extension } \\
/ \mathrm{mm}\end{array}$ & $\begin{array}{c}\text { Experimental } \\
\text { time } / \mathrm{s}\end{array}$ \\
\hline $2^{\text {nd }}$ stage TBS & 0.950 & 0.430 & 23.19 \\
$3^{\text {rd }}$ stage TBS & 1.500 & 0.400 & 39.70 \\
$4^{\text {th }}$ stage TBS & To failure & 0.714 & 41400 \\
Total & & 1.544 & 41462.89 \\
\hline
\end{tabular}

A comparison of the fracture surfaces obtained in both staged creep and preliminary testing are showed in Fig. 7. 

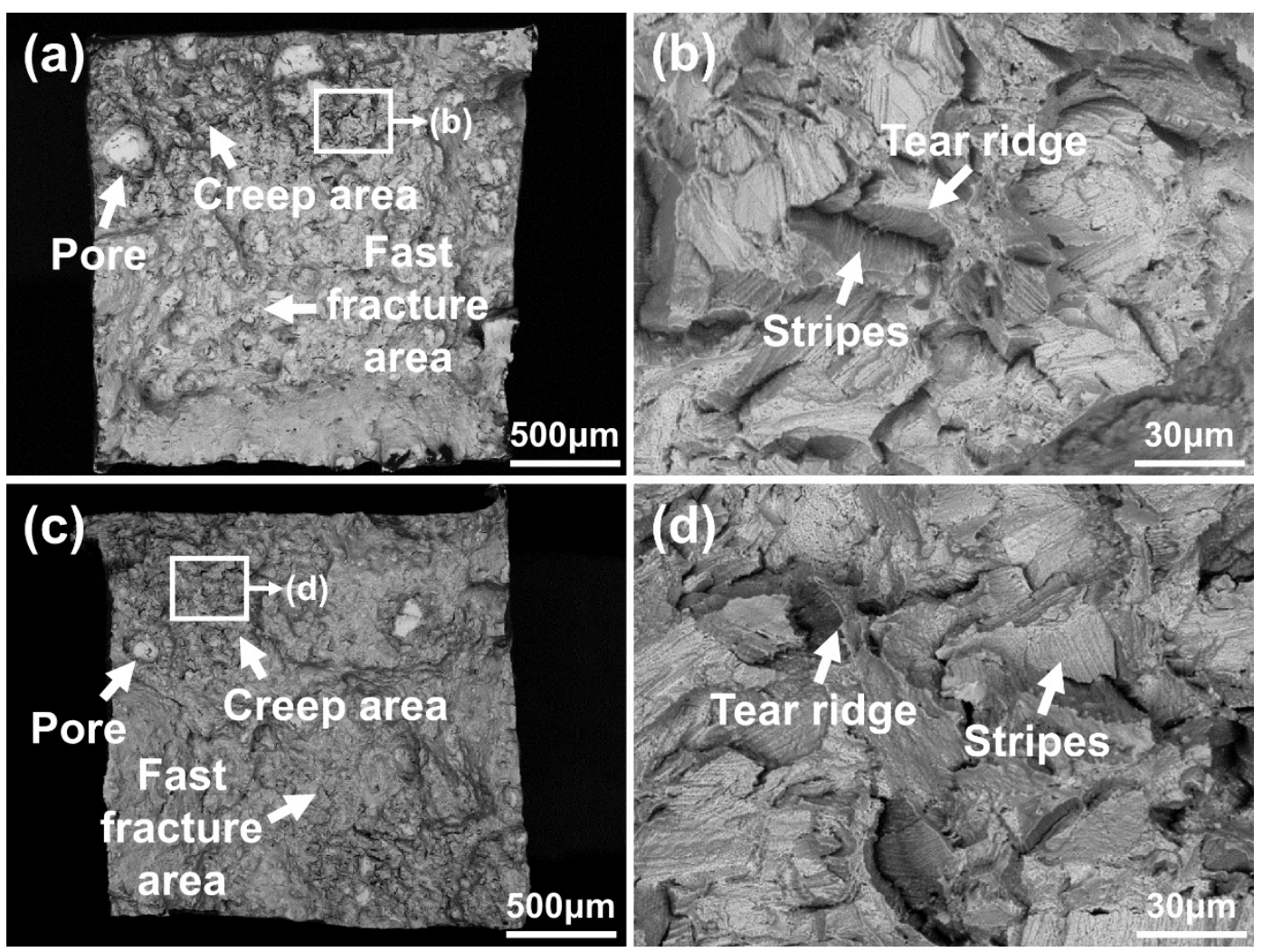

Fig. 7 (a) SEM image of a fracture surface of the staged tested TBS; (b) high magnification of the creep area in image (a); (c) SEM image of a fracture surface of preliminary tested TBS; (d) high magnification of the creep area in image (c).

The two specimens have similar fracture features (such as the tear ridges and striations found in Fig. 7(b) \& (d)), which indicate they failed by a consistent mechanism, and creep is the dominant failure mechanism in the staged testing.

\subsection{XCT measurements}

The cross-sectional area of the TBS specimen was calculated from each image in the image stack during the first three XCT measurement stages (i.e. the $1^{\text {st }}$ stage, $2^{\text {nd }}$ stage and $3^{\text {rd }}$ stage, shown in Fig. 8). The cross-sectional area along the TBS parallel regions decreased notably during creep testing. Also, 'valleys' present at either end of $2^{\text {nd }}$ and $3^{\text {rd }}$ stage data indicate the fracture position in TBS samples. 


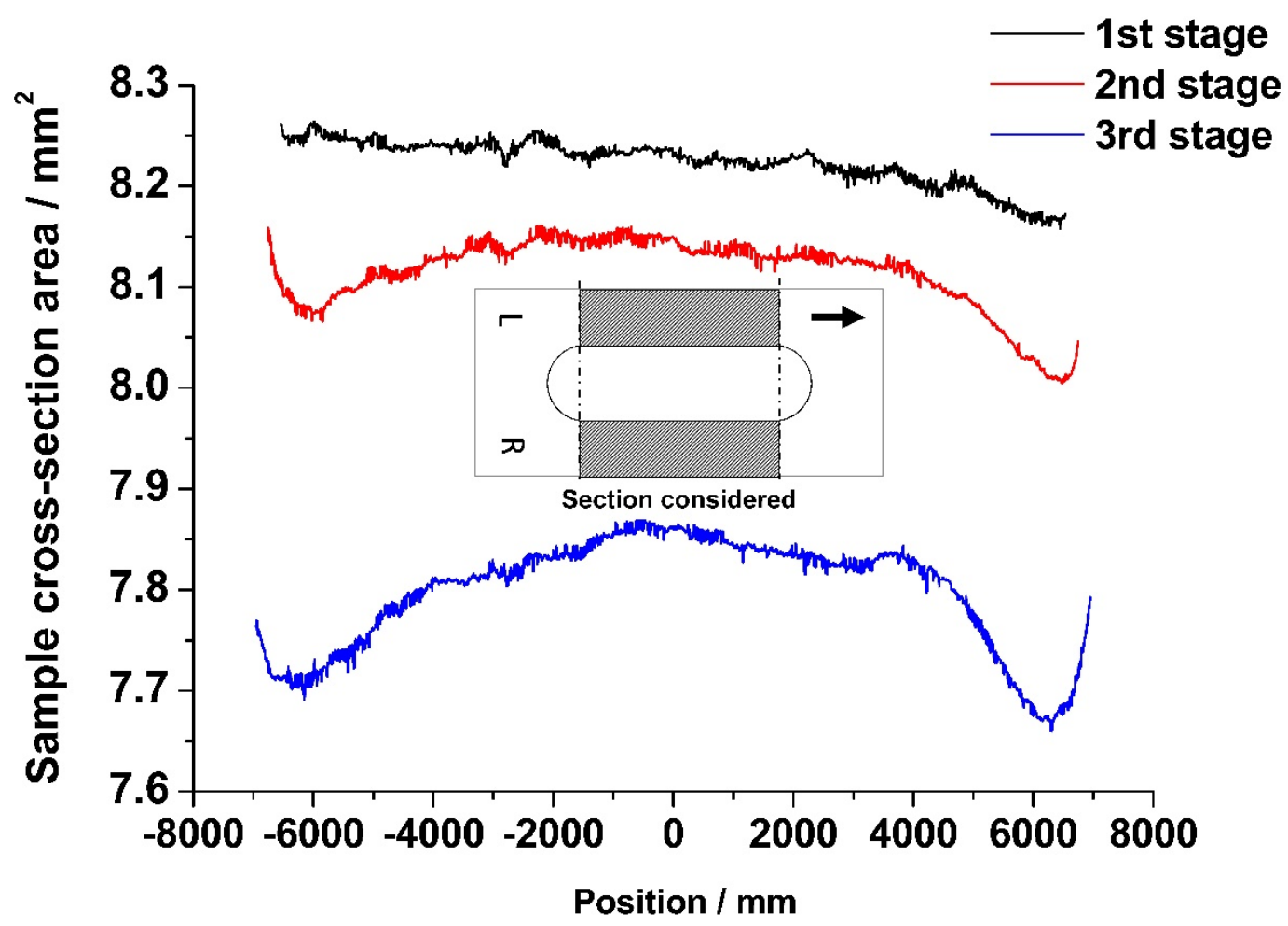

Fig. 8 Using XCT the cross-sectional area as a function of position along the TBS can be computed.

Porosity distributions in the first three stages along the uniaxial section are shown in Fig. 9, where increasing porosity across the three stages, most dramatically in the $3^{\text {rd }}$ stage, can be observed. 

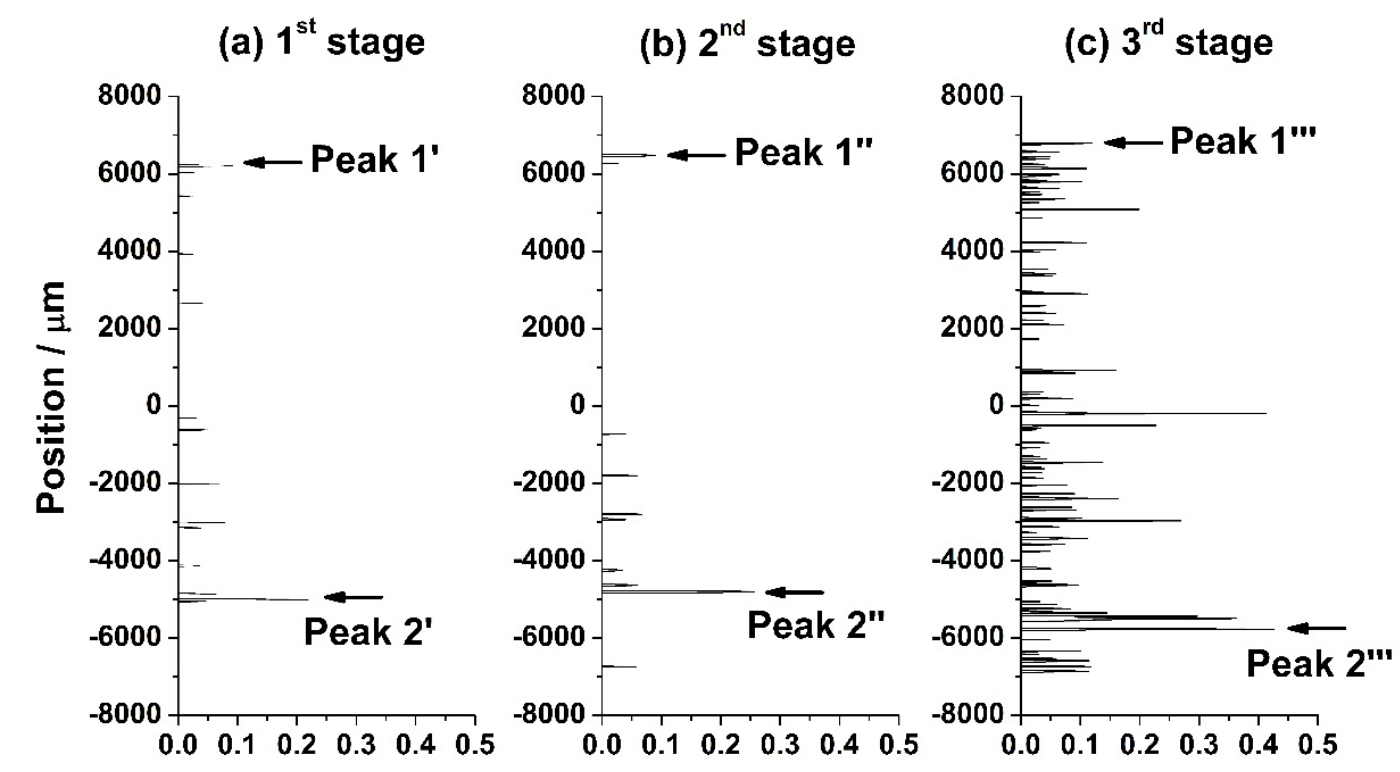

Porosity $/ \%$

Fig. 9 Porosity variation along the building direction includeing both bars of the TBS (a) $1^{\text {st }}$ stage; (b) $2^{\text {nd }}$ stage; (c) $3^{\text {rd }}$ stage.

The porosity and average pore area of the entire parallel region in the first three stages are shown in Fig. 10. The porosity has a $10 \%$ increase between the $1^{\text {st }}$ stage and $2^{\text {nd }}$ stage, and an 8 times increase between $2^{\text {nd }}$ stage and $3^{\text {rd }}$ stage. While the average pore area increased between the $1^{\text {st }}$ and the $2^{\text {nd }}$ stages and then decreased between the $2^{\text {nd }}$ stage and $3^{\text {rd }}$ stages.

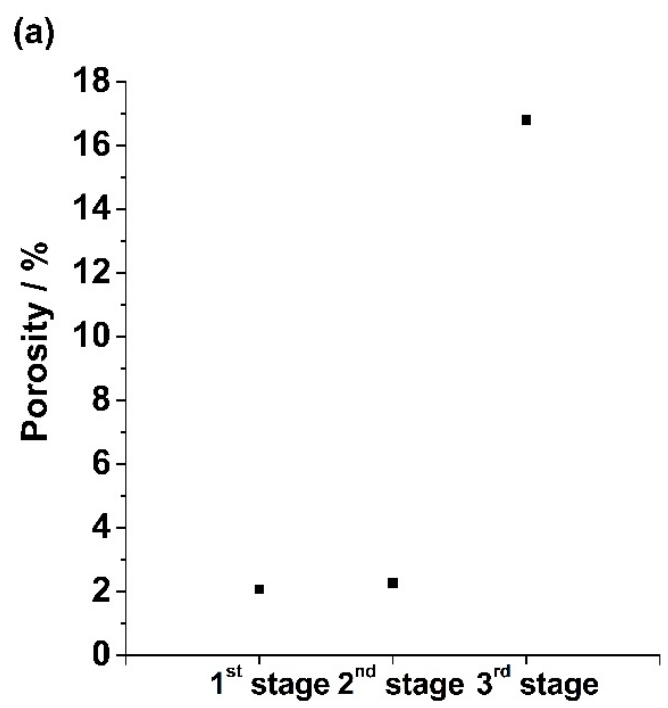

(b)

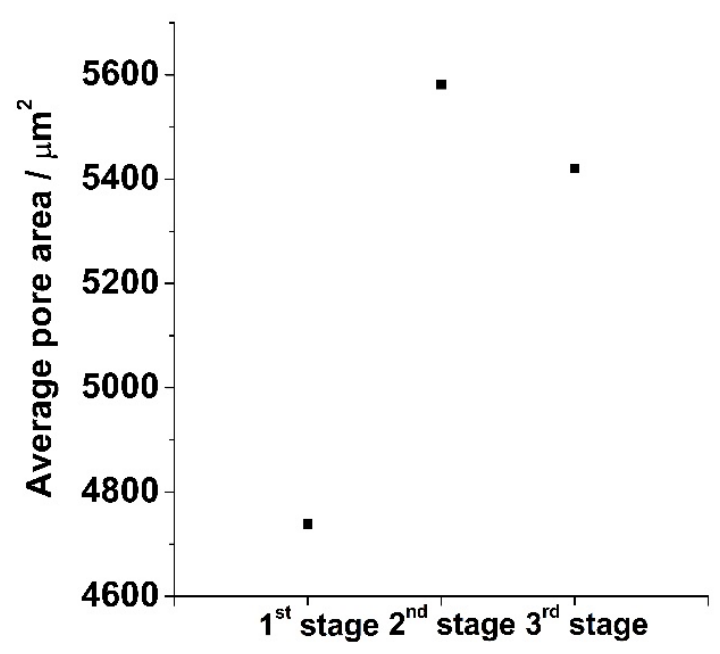

Fig. 10 Porosity comparison at different stages of the staged creep test: (a) porosity; (b) average pore area. 
As shown in Fig. 11, the porosity distribution in the left and right bars of the TBS differs noticeably, likely as a result of the random distribution of the defects appearing during the SLM process.

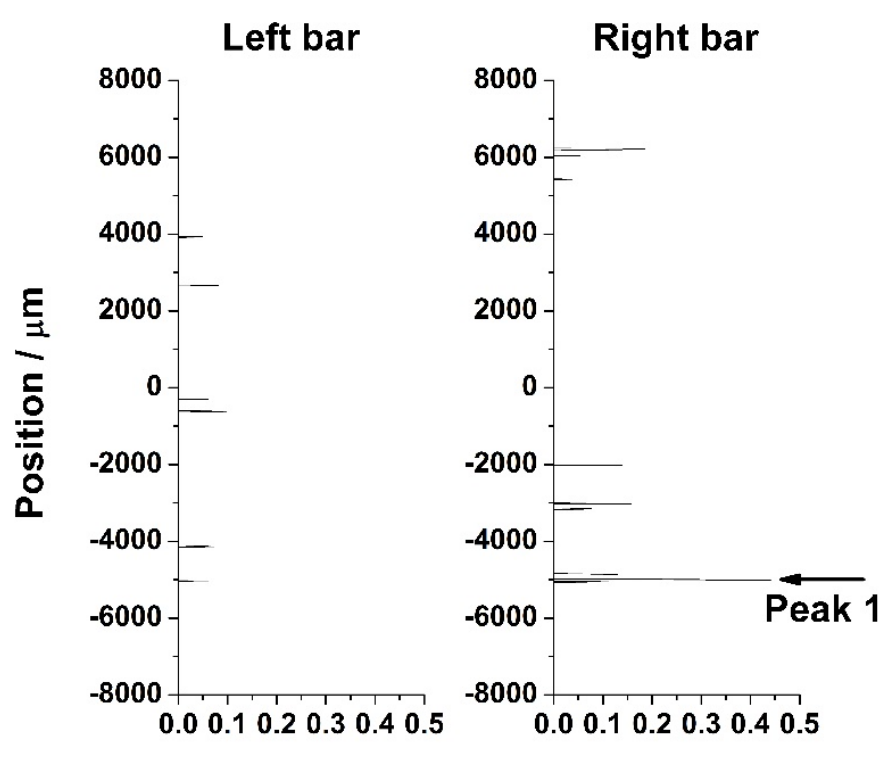

Porosity $/ \%$

Fig. 11 Comparison of the porosity variation along the build direction between the left and right bars of the TBS before creep testing.

By comparing the porosity distribution in the two bars, it is possible to predict that the failure will most likely be observed in the bottom section (marked by Peak 1 in Fig. 11) of the right bar.

\subsection{Fracture analysis}

The failed staged tested TBS was also examined with XCT as shown in Fig. 12, in which the Y-direction is the loading direction. As the left bar was deformed in the $\mathrm{X}$ direction, it is clear that the right bar fractured first. The fracture section was ignored when calculating the porosity distribution since it is difficult to distinguish the pore from the background, as shown in Fig. 12(c), it is also not possible to calculate the crosssectional area of the sample. 

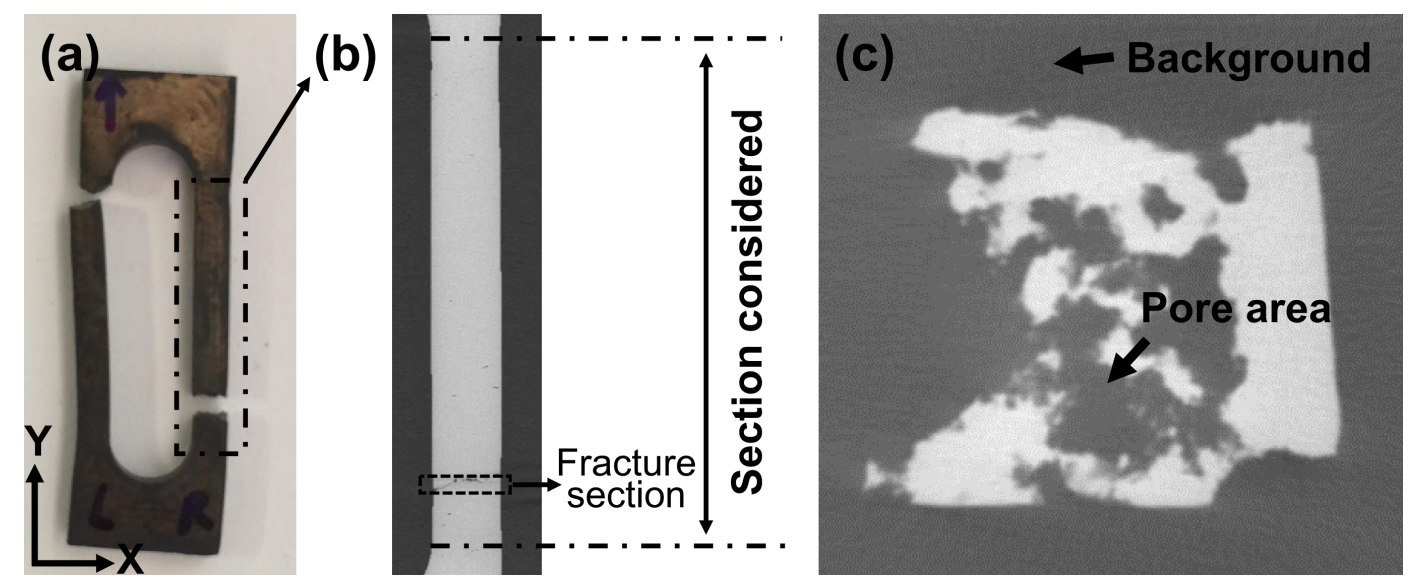

Fig. 12 (a) The fractured TBS; (b) vertical cross-section of the right bar (perpendicular to the uniaxial direction); (c) example cross-section of the fracture section in the uniaxial direction.

By comparing the porosity distribution in all four stages, it is clear that the largest peak present in the first three stages was the cause of the eventual fracture. This peak was no longer observable in the $4^{\text {th }}$ stage, as shown in Fig. 13(d), due to its presence in the fracture section. When examining the XCT data, it is possible to pinpoint the specific pores from which the eventual failure originated. 3D models of the pores in question (as shown in Fig. 14(a), 14(b) and 14(c)) were obtained by assembling XCT photos to provide additional understanding of how the defects in the fracture section (as shown in Fig. 12(b)) evolved during the creep test. Fig. 14(d) shows the SEM image of the interior of the pores which were pinpointed in the $3^{\text {rd }}$ stage, as shown in Fig. 14(c). 


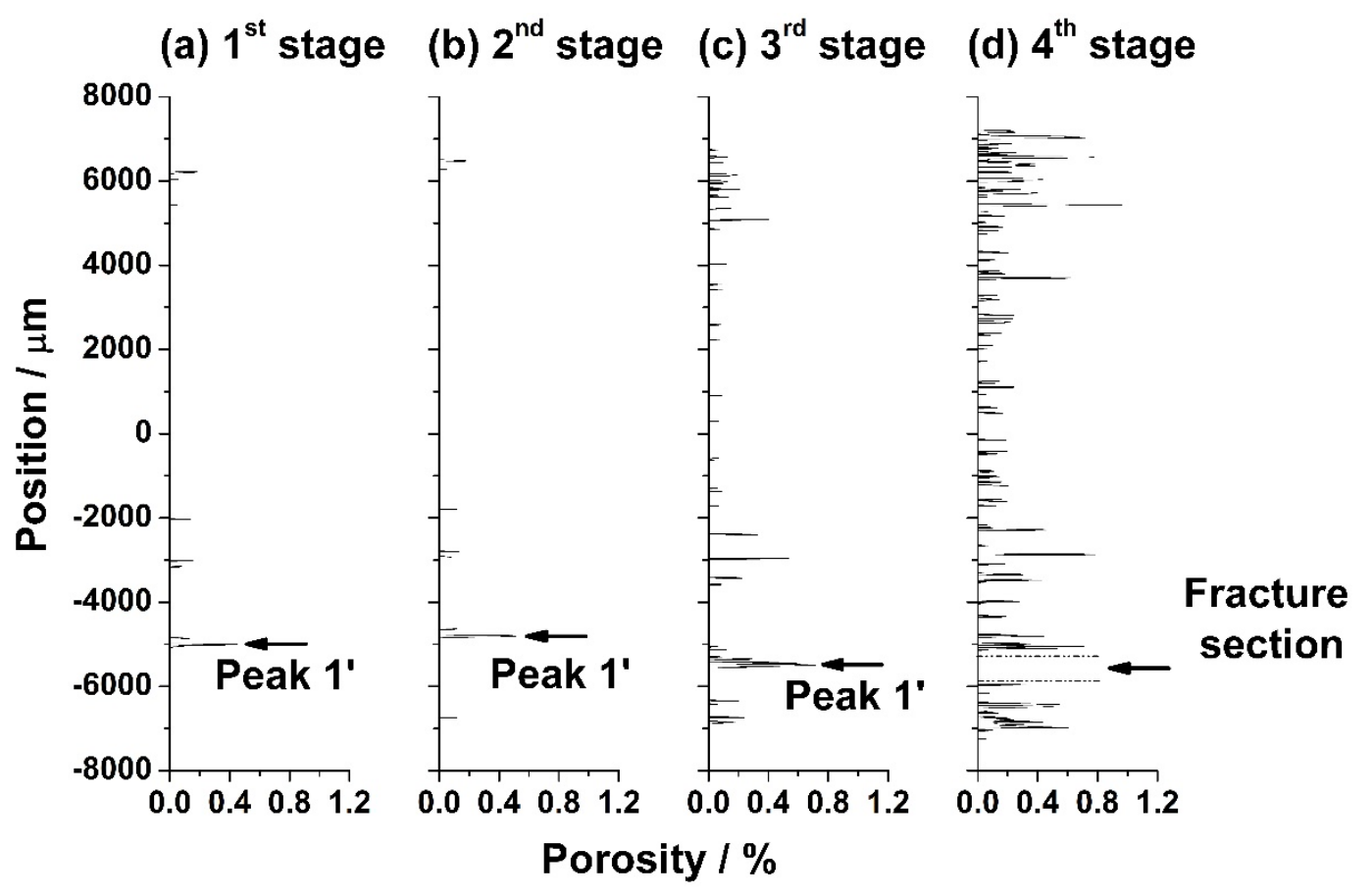

Fig. 13 Porosity variation along the building direction in the right bar: (a) $1^{\text {st }}$ stage; (b) $2^{\text {nd }}$ stage; (c) $3^{\text {rd }}$ stage; (d) $4^{\text {th }}$ stage

(a)

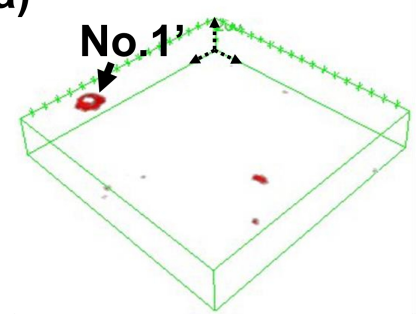

$1^{\text {st }}$ stage

(c)

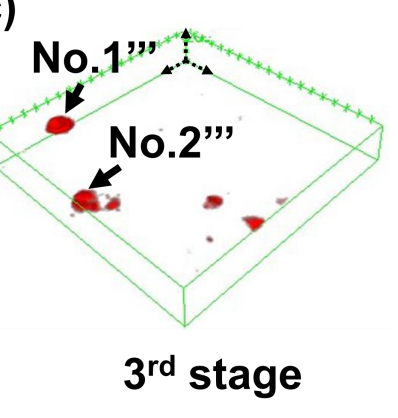

(b)
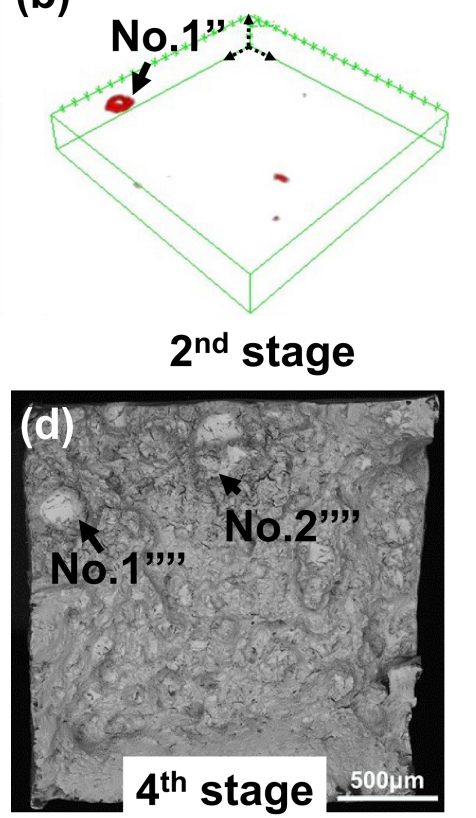

Fig. 14 Visualisations of the evolution of defects in the fracture section in all four stages of the creep test: (a) $1^{\text {st }}$ stage (XCT model); (b) $2^{\text {nd }}$ stage (XCT model); (c) $3^{\text {rd }}$ stage (XCT model); (d) $4^{\text {th }}$ stage (SEM image).

A comparison of the porosity and average pore area among the first three stages was also performed, specifically focussing on the fracture section (as shown in Fig. 12(b)); shown in Fig. 15. 
(a)

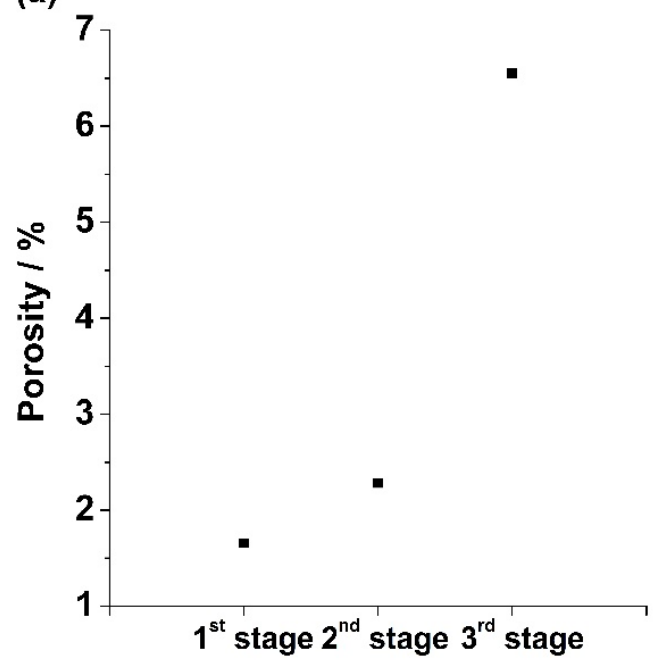

(b)

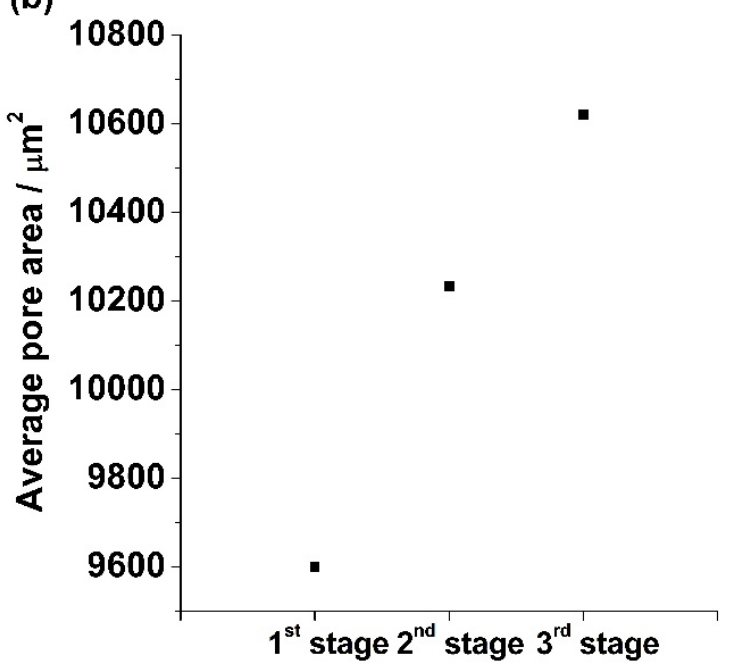

Fig. 15 Porosity comparison in the fracture section at different stages of the staged creep test: (a) porosity; (b) average pore area.

\section{Discussion}

\subsection{Staged creep testing}

As shown in Table 2, the short experimental time for the $2^{\text {nd }}$ stage and $3^{\text {rd }}$ stage indicates that a large proportion of the extension observed was a tensile extension and may still be in the elastic regime. Thus, extensions were smaller than expected. However, the fracture mechanisms and the lifetime of the interrupted and uninterrupted tests show similarities. The fracture surface of staged tested sample shows a creep dominated failure mechanism (as shown in Fig. 7), which is similar to that found in the uninterrupted test. The $2^{\text {nd }}$ and $3^{\text {rd }}$ stage testing may indicate work hardening on the TBS and cause the sample to become more creep resistant, resulting in the lifetime of the crept TBS in this study being higher than the average obtained in the preliminary testing. In additional experimentation it may be reasonable to separate the stages by time, rather than extension, should the testing be repeated, and allow the creep mechanism to be fully developed before the first stage interruption to the test. In general, the accurate determination of the interruption intervals requires a large number of preliminary tests, so that all stages can perform as expected. 


\section{2 $\underline{\text { XCT measurement results }}$}

Firstly, the XCT measurement results provide direct evidence of the SLM process generating randomly distributed defects, with the total porosity of the right bar being $11 \%$ higher than that of the left bar. These inbuilt defects are likely as a result of a combination of both the scanning strategy and the part geometry. Particularly, in the porosity distribution of the right bar, there are some dominant peaks which indicate the weakness of the sample and may act as fracture initiation points in the subsequent testing.

With regard to the entire uniaxial section, an obvious decrease in the cross-sectional area was observed as shown in Fig. 8. While in the tested TBS, the two ends of the TBS were found to have higher area reduction compared to the mid-section, indicating that these two regions are the most porous sections in the tested sample. Therefore the fracture is more likely to happen in the two ends. As can be seen in Fig. 8, the two ends have $30 \%$ more area reduction compared to the midpoint in the $3^{\text {rd }}$ stage when taking the non-crept sample as a benchmark.

Apart from the changes in the cross-sectional area, the porosity distributions are also show the exaggeration of the defects in the TBS. Some porosity peaks (e.g. the peaks marked in Fig. 9) are recognised and quantified (as shown in Fig. 16). Peak 1 grows much faster than Peak 2, thus it has a higher possibility to cause fracture than Peak 2. There are also some new peaks which appeared in the curves which indicate the porosity growth in these regions. 


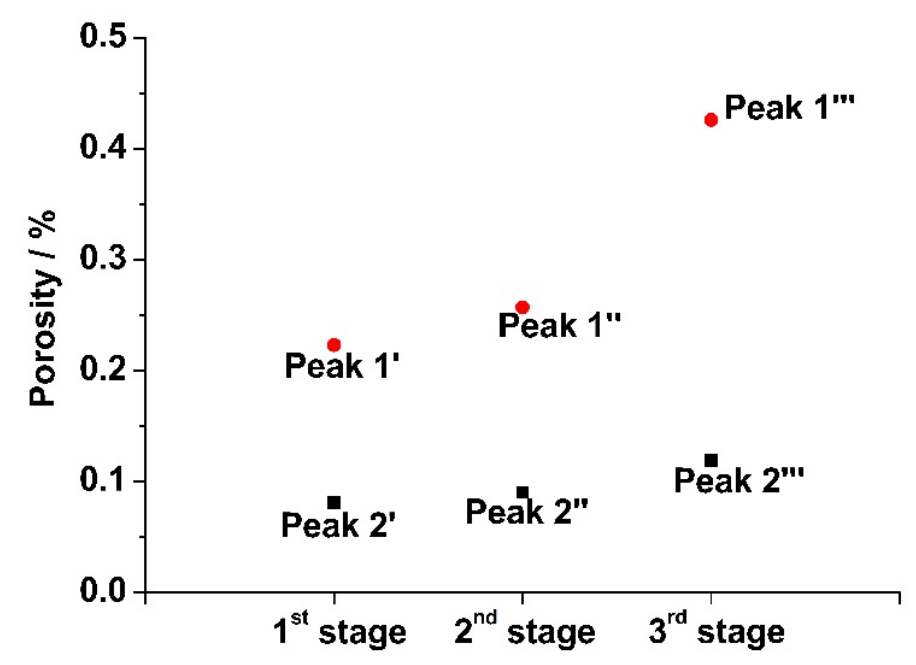

Fig. 16 The porosity increasing among the peaks marked in Fig. 11 as a result of tensile loading

The $3^{\text {rd }}$ stage showed the highest porosity that indicates it is much closer to failure when compared to the first two stages. As shown in the Fig. 10, the $3^{\text {rd }}$ stage has a dramatic degradation compared to the $2^{\text {nd }}$ stage since the porosity in the $3^{\text {rd }}$ stage increased by more than 6 times compared to that in the $2^{\text {nd }}$ stage. While the growth of the number of pores seems play a more important role than the growth of the pore size in the later stage, so that the average pore area in the $3^{\text {rd }}$ stage decreased by $3 \%$.

In this study, a thresholding method was used to recognise the pores. However, in some XCT images, noises were generated simultaneously when trying to pick out the pores with thresholding. Due to a large amount of XCT images, it is not able to verify the thresholding results sequentially. So, inevitably, error rose.

\subsection{Fracture section analysis}

By analysing the porosity distribution of the first broken sample, i.e. the right bar, the origin of the fractures can be pinpointed. Since it is not possible to calculate the porosity in the fracture section immediately before failure, its porosity distribution is not shown in Fig. 13(d). It is also noted that the highest peak observed in the first three stages can no longer be observed in the $4^{\text {th }}$ stage due to the fracture occurring in this region. Thus the defects located in the positions correspond to the highest peaks and play important roles in the onset of fracture. The 3D models are shown in Fig. 14 and clearly show the evolution of the defects in the fracture section. Pore No.1 is observed to grow during the creep testing and a group of new pores generated in the $3^{\text {rd }}$ stage 
TBS as shown in Fig. 14(c). Pore No.1 and newly developed pore No.2 are shown in the fracture surface (as shown in Fig. 14(d)), which indicate these pores may be the origin of the failure. As shown in Fig. 15, both porosity and average pore area in the fracture section are increase during creep. The porosity in the $3^{\text {rd }}$ stage is increased by 1.87 times compared to that in the $2^{\text {nd }}$ stage. The average pore size in the $3^{\text {rd }}$ stage is increased by $3.8 \%$ compared to the $2^{\text {nd }}$ stage, which has an opposite trend compared to the result (as shown in Fig. 10(b)) of the entire considered section. This is a sign that the growth of pore size plays a more important role than the growth of pore number in the fracture section.

\section{Conclusions}

By applying a novel staged creep testing technique together with XCT, TBS performance during the testing was classified. The spatial porosity distributions in all stages were demonstrated. The changes in the porosity and average pore volume in each stage of the testing were quantified and compared. In addition, the origin of the fracture was successfully predicted.

The comparison of the porosity distribution between the two bars in the $1^{\text {st }}$ stage indicates the defects are randomly distributed in the SLM made sample. The total porosity of the right bar is $11 \%$ higher than that of the left bar. In the position marked by peak 1 in Fig. 11, the porosity in the right bar is nine times larger than that in the left bar.

The most porous points were located in the two ends of the parallel region of the sample. The area of the cross-section decreased significantly in these positions during the testing. As a whole, the growth of the pore size is more obvious than the increase of pore numbers at the beginning of the creep testing. In the later stage, the increase of pore numbers become more dominant. While in the fracture section, the growth of pore size is still more dominant in the later stage.

The experimental time of the first two tests is too short time to contribute to fully developed creep mechanisms. It is more reasonable to separate the stages by time which allow the creep mechanism to fully develop in each stage. The thresholding method needs to be optimised to eliminate most of the noise and achieve enhanced 
pore recognition. Other edge detection techniques (to recognise the pores, such as Canny, Sobel, etc. [19]) should also be considered in the future.

\section{Acknowledgements}

The authors would like to acknowledge the support from Engineering and Physical Sciences Research Council (EPSRC Grants EP/L017121/1). In addition, the authors would like to thank Alexander Jackson-Crisp for his invaluable technical contributions in machining specimens, Shane Maskill for facilitating the creep testing and to Renishaw Plc, process engineering team for providing Inconel 718 samples.

\section{Reference}

[1] K.N. Amato, S.M. Gaytan, L.E. Murr, E. Martinez, P.W. Shindo, J. Hernandez, S. Collins, F. Medina, Microstructures and mechanical behavior of Inconel 718 fabricated by selective laser melting, Acta Materialia 60(5) (2012) 2229-2239.

[2] Q. Jia, D. Gu, Selective laser melting additive manufacturing of Inconel 718 superalloy parts: Densification, microstructure and properties, Journal of Alloys and Compounds 585(0) (2014) 713-721.

[3] L. Rickenbacher, T. Etter, S. Hövel, K. Wegener, High temperature material properties of IN738LC processed by selective laser melting (SLM) technology, Rapid Prototyping Journal 19(4) (2013) 282-290.

[4] M. Pröbstle, S. Neumeier, J. Hopfenmüller, L.P. Freund, T. Niendorf, D. Schwarze, M. Göken, Superior creep strength of a nickel-based superalloy produced by selective laser melting, Materials Science and Engineering: A 674 (2016) 299-307.

[5] S.F. Diltemiz, S. Zhang, Superalloys for super jobs, in: S. Zhang, D. Zhao (Eds.), Aerospace Material Handbook, CRC Press, London, 2013, pp. 1-76.

[6] W. Sun, S.B. Beown, R.K. Leach, An Overview of Industrial X-ray Computed Tomography, National Physical Laboratory, London, 2012.

[7] Y. Hangai, O. Kuwazuru, T. Yano, T. Utsunomiya, Y. Murata, S. Kitahara, S. Bidhar, N. Yoshikawa, Clustered Shrinkage Pores in Ill-Conditioned Aluminum Alloy Die Castings, MATERIALS TRANSACTIONS 51(9) (2010) 1574-1580.

[8] L. Babout, E. Maire, J.Y. Buffière, R. Fougères, Characterization by X-ray computed tomography of decohesion, porosity growth and coalescence in model metal matrix composites, Acta Materialia 49(11) (2001) 2055-2063.

[9] S. Terzi, L. Salvo, M. Suéry, N. Limodin, J. Adrien, E. Maire, Y. Pannier, M. Bornert, D. Bernard, M. Felberbaum, M. Rappaz, E. Boller, In situ X-ray tomography 
observation of inhomogeneous deformation in semi-solid aluminium alloys, Scripta Materialia 61(5) (2009) 449-452.

[10] A. Thompson, I. Maskery, R.K. Leach, X-ray computed tomography for additive manufacturing: a review, Measurement Science and Technology 27(7) (2016) 072001.

[11] I. Maskery, N.T. Aboulkhair, M.R. Corfield, C. Tuck, A.T. Clare, R.K. Leach, R.D. Wildman, I.A. Ashcroft, R.J.M. Hague, Quantification and characterisation of porosity in selectively laser melted Al-Si10-Mg using X-ray computed tomography, Materials Characterization 111 (2016) 193-204.

[12] S. Tammas-Williams, H. Zhao, F. Léonard, F. Derguti, I. Todd, P.B. Prangnell, XCT analysis of the influence of melt strategies on defect population in Ti-6Al-4V components manufactured by Selective Electron Beam Melting, Materials Characterization 102 (2015) 47-61.

[13] T.H. Hyde, B.S.M. Ali, W. Sun, Analysis and Design of a Small, Two-Bar Creep Test Specimen, Journal of Engineering Materials and Technology 135(4) (2013) 041006-041006.

[14] T. Sugahara, K. Martinolli, D. A.P. Reis, C. de Moura Neto, A. A. Couto, F. P. Neto, M.J.R. Barboza, Creep Behavior of the Inconel 718 Superalloy, Defect and Diffusion Forum 326-328 (2012) 509-514.

[15] H. Jiang, Image reconstruction, Computed Tomography Principles, Design, Artifacts, and Recent Advances, SPIE Press, Bellingham, USA, 2009.

[16] O. Marques, Image segmentation, Practical Image and Video Processing Using MATLAB, Wiley, Hoboken, New Jersey, 2011, pp. 368-370.

[17] C.A. Schneider, W.S. Rasband, K.W. Eliceiri, NIH Image to ImageJ: 25 years of image analysis, Nat Meth 9(7) (2012) 671-675.

[18] J.P. Kruth, M. Bartscher, S. Carmignato, R. Schmitt, L. De Chiffre, A. Weckenmann, Computed tomography for dimensional metrology, CIRP Annals Manufacturing Technology 60(2) (2011) 821-842.

[19] O. Marques, EDGE DETECTION, Practical Image and Video Processing Using MATLAB, Wiley, Hoboken, New Jersey, 2011, p. 335. 